\title{
TEACHING UNDERGRADUATES MICROSCOPY FOR THIRTY YEARS: LESSONS LEARNED
}

\section{Robert V. Blystone}

\section{Department of Biology, Trinity University, San Antonio, Texas 78212, USA}

On August 13, 1973, a paper titled "Visual aids in electron microcopy instruction" was presented at the education section of the EMSA annual meeting in New Orleans, Louisiana. The paper described teaching Trinity University undergraduate students transmission electron microscopy (TEM) within the context of a regular techniques class. In 1978 the National Science Foundation awarded grant SER 78-13132, Instructional Scientific Equipment Program, to Trinity University. The purpose of the grant to was to add a scanning electron microscope (SEM) to the two transmission electron microscopes being used to teach undergraduates electron microscopy. In August of 1989 EMSA met in San Antonio, Texas, and I followed John Mansfield and Nestor Zaluzec about the convention exhibit floor. I watched them put together a local area network on the floor and use NIH Image to process images obtained from working equipment to the absolute amazement of the vendors. In 1991 the National Science Foundation awarded grant USE 9152675, Instrumentation and Laboratory Improvement Program, to Trinity University. The grant was titled "A Microcomputer Visualization Laboratory for the Biological Sciences" and resulted in digital imaging optical microscopy being used in the undergraduate laboratory. After 25 years of teaching a total of 278 undergraduates electron microscopy, the three electron microscopes were dismantled and removed in 1996. In 1997 a new course titled "Biological Visualization" was incorporated into the undergraduate curriculum. In 2003, the original digital imaging laboratory was dismantled and replaced after 13 years. In August of 2003 I am giving a paper dealing with teaching digital microscopy in the undergraduate environment.

Initially (1971) the goal was to teach the technique of TEM. A techniques course was devised that was project- and problem-based. As the students learned the technique, they addressed problems using the recently acquired knowledge of the technique. There were 15 or so undergraduates in the semester-long course that was taught annually. Approximately 30 students practiced electron microscopy after graduation with about ten graduates staying in the field ten years or more. A prominent feature of the course was micrograph analysis; students were given an electron micrograph and each had to write an extensive analysis of the meaning associated with the photomicrograph. The effort was largely descriptive, two-dimensional, and library oriented. It was for many students an exercise in technical writing.

As optical microscopy regained prominence and desktop computers with associated software allowed quantitative exploration of images, the nature of undergraduate training in this area shifted dramatically. Rather than focusing on how to produce an image, the goal became working with the image. My approach to teaching microscopy abruptly changed after being introduced to image analysis by Mansfield and Zaluzec in 1989. Aided by several seminars given by John Russ and armed with Russ's textbook and the public domain NIH-Image software, I inserted digital optical microscopy into two regular undergraduate courses: Microanatomy (histology) and Developmental Biology (embryology). In the early 1990's students were extremely computer naïve and software such as Adobe Photoshop and Microsoft PowerPoint 
were foreign to the students. I quickly become known as a "computer jockey" as opposed to a biologist. As the decade progressed students became more familiar with computer use and I could again focus on the analysis of biological information.

Lab work in these courses incorporate significant (but not exclusive) amounts of digital imaging. I stress several principles: 1) the image is numerical data; 2) microscopy serves to collect energy after it interacts with the sample; 3) the data can be expressed in 3, 4, or even 5 dimensions; and 4) and JPEG and TIFF files are not the same thing. Early on I decided to use standard software such as Adobe Photoshop rather than to write code. John Russ's Image Processing Cookbook software facilitated this decision. Digital optical microscopy allowed me to do the following with the undergraduate microscope-based course: establish Digital Legacies; convert static lab practicals to student produced PowerPoint lab exams; establish case studies as part of lab; give students a three-dimensional command of the microanatomy; and changing descriptive microscopy labs into inquiry-based labs. Each class left libraries of images upon which the next class could build. Students moved beyond a single slide sample as student produced CD's and Web image sources could provide an in-depth sample of structures. Students could respond to quantitative-based questions rather than identify the structure at the end of the pointer. Being able to framegrab lab work encouraged the students to personally claim the image and its associated knowledge as their own.

The most interesting aspect of the coursework became the problem solving and visualization. Two notable efforts were the three-dimensional analysis of lacunae distribution in compact bone and the three-dimensional reconstruction of the cranial nerves of a 72-hour chick embryo. Over a period of several years successive classes reworked the exercises with increasing sophistication. From the simple morphing of the events of mitosis to modeling volume changes in cleaving sea urchin embryos, the students rose to the occasion and opportunities presented by digital imaging. By having students make proactive presentations of their knowledge of histological structures, I can press for mastery of the image rather than getting a 70 on the practical exam.

A major impetus for moving to digital optical microscopy was to avoid the cost of maintaining an electron microscopy lab. In thirteen years, however, the equipment for the digital microscopy lab has been cycled four times. Storage media change, bus connections change, and networking protocols change. Keeping software current and legal are major challenges. Keeping digital equipment current has been more difficult than maintaining the TEM lab equipment twenty years ago. Another unforeseen challenge has come from the administration. In the past my purchase requests for optical equipment were fulfilled promptly and without question (especially if I provide the funding). However, administrators are constantly challenging my computer hardware and operating system choices (even with extramural funding). They view the computer as separate from digital imaging. The presentation based on the above experiences will explore the work of the students over the years and how digital optical imaging has led to dramatic changes in the questions that can be posed in lab and lecture. 